\title{
From an ACE polymorphism to genome-wide searches for $\mathrm{eQTL}$
}

\author{
Florent Soubrier
}

Genetics Department, Hospital Pitié-Salpêtrière, Assistance Publique-Hôpitaux de Paris, and UMR_S 956 UPMC-INSERM, Paris, France.

\begin{abstract}
Angiotensin I-converting enzyme (ACE, or DCP1) is a zinc metallopeptidase that converts angiotensin $I$ into the vasoactive and aldosterone-stimulating peptide angiotensin II and cleaves bradykinin into inactive peptides. Plasma ACE measurement is widely used for the diagnosis of sarcoidosis. While enzyme concentrations are highly stable in an individual, there is a high level of interindividual variability. In 1990, we identified an insertion/deletion polymorphism in $A C E$ that functions as a quantitative trait locus (QTL), accounting for half of the interindividual variability. Since then, technological advances have allowed for the elucidation of expression QTLs (eQTL). Such studies are allowing researchers to determine how underlying genetic predisposition contributes to human disease.
\end{abstract}

Angiotensin I-converting enzyme (ACE, or DCP1) plays a major role in the renin angiotensin system (RAS) by converting an inactive decapeptide, angiotensin I, into the vasoactive and growth promoting octapeptide angiotensin II (AII), and also by degrading bradykinin into inactive peptides $(1,2)$. Indeed, AII is the main active peptide both of the circulating RAS, which has hemodynamic effects through vasoconstriction and sodium retention, and of the tissue RAS, which regulates microcirculation in organs such as the heart or the kidney. AII increases cell proliferation, ROS production, cell growth, migration and differentiation, and extracellular matrix remodeling, and is proinflammatory and profibrotic (3). AII can lead to tissue injury by activating multiple intracellular signaling pathways. The beneficial effects of ACE inhibitors on renal function during diabetes and on heart function in ischemic or failing hearts have been demonstrated across several studies (4). Bradykinin is a vasodilator and natriuretic peptide that antagonizes angiotensin-induced vasoconstriction and sodium retention (5). Should genetic variation in the ACE gene modify its ability to metabolize vasoactive peptides, it could be considered a candidate gene for a predisposition to develop various cardiovascular and kidney diseases.

Conflict of interest: The author has declared that no conflict of interest exists.

Citation for this article: J Clin Invest. 2013; 123(1):111-112. doi:10.1172/JCI66618.
After we determined the amino acid structure of ACE by cloning its CDNA, we used the ACE cDNA probe as a genetic tool for exploring both the ACE plasma level polymorphism and the potential role of the gene in the predisposition to cardiovascular and renal diseases. The JCI article by Rigat et al. that we comment on here showed a clear relationship between an ACE marker genotype consisting of two different alleles, the insertion (I) and the deletion (D) allele within intron 16 of the gene, and the serum level of the enzyme, which is known to be stable within a given individual, but to differ among individuals (6). A previous segregation study in healthy families by Cambien et al. showed that ACE serum levels were under the influence of a "major gene," also called a quantitative trait locus (QTL) (7). Using a DNA probe for the ACE gene (8), we identified the genetic locus underpinning the observed QTL, demonstrating that an ACE gene polymorphism was acting in cis on the serum ACE level, as well as on the ACE mRNA level (9). This strong relationship has been replicated several times since.

Since the polymorphic insertion is a repetitive element within an intron of $A C E$, we were keen to determine whether this I/D polymorphism was itself responsible for the expression variation in ACE or whether it was only a marker in linkage disequilibrium with functional variants. Using additional ACE markers in 96 families, we showed that there were indeed two QTL within the ACE gene, one located in the $5^{\prime}$ region, and another one in the cen- tral part of the gene (10). These results were confirmed in a later study, which included an extensive search for $A C E$ gene variants, with subsequent genotyping of these variants in a larger series of 250 healthy families of mixed European descent. Clade analysis of the haplotypes was then performed in order to search for associations with ACE serum levels (11). Two recombination break points were detected in the major haplotypes, one upstream of the transcription initiation site and the other in the $3^{\prime}$ region flanking the $A C E$ gene. The major effect was due to variants located between these two recombination points, and a weaker effect was linked to the upstream region. Indeed, the high-linkage disequilibrium observed between the markers in European subjects prevented further identification of the functional variant. Greater haplotype diversity was observed in West African families, resulting in the exclusion of the I/D polymorphism as the functional variant. These results indicated that multiple sites influence circulating ACE levels, among which four potential sites were candidates for a direct effect (12). Further in vitro studies with cloned gene fragments could not confirm the functional role of these variants (our unpublished results). Thus, 20 years after its initial publication, the molecular basis underlying the expression QTL (eQTL) of the ACE gene has not been elucidated at the DNA level.

Such a relationship involving a major $R A S$ gene spurred numerous association studies focused on discovering the links between the ACE eQTL and a genetic risk for cardiovascular or renal diseases. Results of case-control studies are often conflicting and prone to errors due to recruitment bias, population stratification, and multiple comparisons, requiring validation in independent groups of subjects. The hundreds of studies that have taken place since the identification of the ACE I/D polymorphism have all aimed to discover associations between the ACE I/D marker and various diseases involving the renin angiotensin or kal- 
likrein kinin systems, such as myocardial infarction or coronary heart disease, stroke, renal diseases, or complications of diabetes (13-17). Study results were generally conflicting, but a recent metaanalysis of 50 case-control studies on the ACE I/D polymorphism and stroke concluded that carrying the ACE D allele constituted a low penetrance risk factor (18). Indeed, meta-analyses conducted with rigorous statistical methodology will help to clarify any associated risk with the $A C E$ gene polymorphism, although a bias toward the publication of positive results is possible.

Genetically engineered mice carrying either a single copy, or two or three active copies of the ACE gene were generated in the Smithies laboratory, allowing the effects of increased ACE expression to be tested in different pathological models (19). Indeed, three-copy mice presented with increased proteinuria after 12 weeks of diabetes, as compared with mice carrying only one or two copies (20). ACE mRNA was increased in the kidney of three-copy mice, and decreased bradykinin levels were proposed to account for the marked effects on glomerular hemodynamics during diabetes in these mice. This experimental model combining genetically increased ACE and diabetes represents a valuable support for the effect of the ACE I/D polymorphism on diabetes complication in humans (21).

The ACE gene represents a paradigm of eQTL, and within 20 years, a complete revolution has occurred. eQTL searches have benefited from the "omics" revolution, combining genome-wide SNP genotyping, transcript expression analysis via microarray or RNAseq, and determination of thousands of protein levels in biological fluids by proteomic analysis (22). Combining these measurements allows a genome-wide search for eQTL that are relevant to disease susceptibility, such as the study performed by Cham- bers et al. for the plasma concentration of liver enzymes (23). These eQTL are essential for understanding the associations between SNPs and complex diseases by genome-wide association studies. By identifying a growing number of these relationships, we will be able to put together, like pieces of a puzzle, the elementary traits underlying genetic predisposition to cardiovascular diseases.

\section{Acknowledgments}

I thank Pierre Corvol for critical reading of the manuscript, and Rachel Peat for kindly editing the manuscript.

Address correspondence to: Florent Soubrier, Genetics Department, Hospital PitiéSalpêtrière, Assistance Publique-Hôpitaux de Paris, and UMR_S 956 UPMC-INSERM. 91 bvd de l'hôpital, 75634 PARIS cedex 13. Phone: 33.1.40779743; Fax: 33.1.40779645; E-mail: florent.soubrier@upmc.fr.

1. Ehlers MR, Riordan JF. Angiotensin-converting enzyme: new concepts concerning its biological role. Biochemistry. 1989;28(13):5311-5318.

2. Corvol P, Williams TA, Soubrier F. Peptidyl dipeptidase A: Angiotensin I-converting enzyme. Methods Enzymol. 1995;248:283-305.

3. Rüster C, Wolf G. Renin-angiotensin-aldosterone system and progression of renal disease. J Am Soc Nephrol. 2006;17(11):2985-2991.

4. Azizi M, Ménard J. Combined blockade of the renin-angiotensin system with angiotensinconverting enzyme inhibitors and angiotensin II type 1 receptor antagonists. Circulation. 2004;109(21):2492-2499.

5. Madeddu P, Emanueli C, El-Dahr S. Mechanisms of disease: the tissue kallikrein-kinin system in hypertension and vascular remodeling. Nat Clin Pract Nephrol. 2007;3(4):208-221.

6. Rigat B, Hubert C, Alhenc-Gelas F, Cambien F, Corvol P, Soubrier F. An insertion/deletion polymorphism in the angiotensin-I-converting enzyme gene accounting for half the variance of serum enzyme levels. J Clin Invest. 1990;86(4):1343-1346.

7. Cambien F, et al. Familial resemblance of plasma angiotensin-converting enzyme level: The Nancy Study. Am J Hum Genet. 1988;43(5):774-780.

8 . Soubrier F, et al. Two putative active centers in human angiotensin I-converting enzyme revealed by molecular cloning. Proc Natl Acad Sci USA. 1988;85(24):9386-9390

9. Costerousse O, Allegrini J, Lopez M, Alhenc-Gelas
F. Angiotensin I-converting enzyme in human circulating mononuclear cells: genetic polymorphism of expression in T-lymphocytes. Biochem J. 1993;290(pt 1):33-40.

10. Villard E, Tiret L, Visvikis S, Rakotovao R, Cambien F, Soubrier F. Identification of new polymorphisms of the angiotensin I-converting enzyme (ACE) gene, and study of their relationship to plasma ACE levels by two-QTL segregation-linkage analysis. Am J Hum Genet. 1996;58(6):1268-1278.

11. Soubrier F, et al. High-resolution genetic mapping of the ACE-linked QTL influencing circulating ACE activity. EurJ Hum Genet. 2002;10(9):553-561.

12. Cox R, et al. Angiotensin-1-converting enzyme (ACE) plasma concentration is influenced by multiple ACE-linked quantitative trait nucleotides. Hum Mol Genet. 2002;11(23):2969-2977.

13. Cambien F, et al. Deletion polymorphism in the gene for angiotensin-converting enzyme is a potent risk factor for myocardial infarction. Nature. 1992;359(6396):641-644

14. Marre M, et al. Relationships between angiotensin I converting enzyme gene polymorphism, plasma levels, and diabetic retinal and renal complications. Diabetes. 1994;43(3):384-388.

15. Lindpaintner $\mathrm{K}$, et al. A prospective evaluation of an angiotensin-converting-enzyme gene polymorphism and the risk of ischemic heart disease. NEngl JMed. 1995;332(11):706-711.

16. Zee RY, Ridker PM, Stampfer MJ, Hennekens CH, Lindpaintner K. Prospective evaluation of the angiotensin-converting enzyme insertion/deletion polymorphism and the risk of stroke. Circulation. 1999;99(3):340-343.

17. Ledru F, et al. Relation between severity of coronary artery disease, left ventricular function and myocardial infarction, and influence of the ACE I/D gene polymorphism. Am J Cardiol. 1998;82(2):160-165.

18. Zhang Z, Xu G, Liu D, Fan X, Zhu W, Liu X. Angiotensin-converting enzyme insertion/deletion polymorphism contributes to ischemic stroke risk: a meta-analysis of 50 case-control studies. PLoS One. 2012;7(10):e46495.

19. Krege JH, et al. Angiotensin-converting enzyme gene mutations, blood pressure, and cardiovascular homeostasis. Hypertension. 1997;29(1 pt 2):150-157.

20. Huang W, et al. Genetically increased angiotensin I-converting enzyme level and renal complications in the diabetic mouse. Proc Natl Acad Sci U S A. 2001;98(23):13330-13334.

21. Hadjadj S, et al. Association between angiotensinconverting enzyme gene polymorphisms and diabetic nephropathy: case-control, haplotype, and family-based study in three European populations. J Am Soc Nephrol. 2007;18(4):1284-1291.

22. Cookson W, Liang L, Abecasis G, Moffatt M, Lathrop M. Mapping complex disease traits with global gene expression. Nat Rev Genet. 2009; 10(3):184-194

23. Chambers JC, et al. Genome-wide association study identifies loci influencing concentrations of liver enzymes in plasma. Nat Genet. 2011; 43(11):1131-1138. 